\title{
Doubly disabled: diabetes in combination with an eating disorder
}

\author{
Anne Ward, Nicholas Troop, Mario Cachia, Peter Watkins, Janet Treasure
}

\begin{abstract}
Summary
We present a series of patients with both an eating disorder and diabetes mellitus and compare these to a group of nondiabetic patients from the same clinic. Significantly more of the diabetic patients had previous attempts at treatment for their eating disorder. A high incidence of diabetic complications was noted with clear implications, both clinically and economically, for early intervention. The incidence of childhood trauma was lower in the diabetic than the non-diabetic group. In the majority of patients, diabetes developed before the eating disorder, suggesting that diabetes itself may provide the vulnerability and increase the risk of developing an eating disorder. Early intervention in diabetic clinics may prevent the development of serious eating disorders.
\end{abstract}

Keywords: diabetes mellitus, eating disorders, liaison psychiatry

Eating disorders and diabetes mellitus are both common conditions, and often coexist. The first case report of a patient with both anorexia nervosa and diabetes only appeared in $1973 .^{1}$ Bulimia nervosa was first described in $1979^{2}$ and, the following year, the second reported association between diabetes and eating disorders appeared. ${ }^{3}$ By 1990, a review of the literature revealed 57 reported cases. ${ }^{4}$

It is controversial whether the prevalence of eating disorders is increased in a diabetic population, with some investigators finding an association, 5,6 and others finding no such increase. $^{7-9}$ There are methodological problems with many of the studies carried out to date, which perhaps explains their conflicting results. Most surveys of clinical populations rely on the use of screening questionnaires, where patients can either avoid participation or 'fake' the answers. All epidemiological surveys in the field of eating disorders have encountered this problem and addressed it in different ways. ${ }^{10-13}$ In one group of patients with infertility problems, a standard instrument, the EAT-26, missed over a quarter of cases. It was common for these women to fear that their eating disorder had 'damaged' them, perhaps contributing to their infertility..$^{14}$ This wish to avoid blame is probably as high among diabetic patients, because of their history of being monitored by professionals. Thus it is possible that many surveys of diabetic populations have underestimated the incidence of eating disorders. On the other hand, earlier screening instruments included items which scored positively by virtue of the patients' correct diabetic practices, and so may have yielded falsepositive results. More recent studies using questionnaires have addressed this, ${ }^{15}$ and in any event it is unlikely to have counterbalanced the low sensitivity discussed above.

Studies using interviews have been smallscale. For example, Peveler et al interviewed a sample of 76 adolescents with diabetes ( 33 girls and 43 boys), and included a non-diabetic control group. They found a higher level of risk factors for bulimia among the diabetic girls, in that they were at a higher weight and a greater proportion were dieting. A third of the female sample admitted to omitting insulin in an effort to control their weight and shape. The levels of glycosylated haemoglobin in the eating disorder sample was $13 \%$; in those who admitted to omitting insulin it was $12.6 \%$, and in the rest of the population, $11.8 \%$. It should be noted that the mean age of the group was only 15, which is below the peak age of onset for eating disorders. Moreover, the sample size was too small to examine whether eating disorders or subclinical cases were more common among the diabetics. Peveler estimated that a four-fold increase in sample size would have been required to detect a two-fold increase in eating disorders in the diabetic population. ${ }^{8}$

The prevalence of bulimia nervosa is not accurately known, hence the need for control groups in these studies. However, a reasonable estimate would be $2 \%$ in the population of young females at risk. ${ }^{16-18}$ On this basis, one could calculate that a population of approximately 1250 diabetic patients would be needed to detect a two-fold increase in bulimia. Clearly, a much larger sample would be needed to study anorexia and diabetes. Therefore, although the prevalence studies to date do not, on balance, support an increased prevalence of eating disorders in diabetes, this may be an artefact of study design. The difficulties of carrying out accurate prevalence studies should not obscure the valuable clinical information available from smaller studies.

What does emerge clearly from the literature is that the combination of diabetes and an eating disorder often results in poorer diabetic control, and a greater risk of serious physical complications. ${ }^{8,9,19,20}$ However, diabetic control 
in women with eating disorders is not necessarily poor and different patterns are observed. ${ }^{21}$ In the case of anorexia nervosa, diabetes may be chronically poorly controlled or 'brittle', and the anorexia may represent another stage in a long history of behavioural disturbance. More commonly, control deteriorates as the patient omits insulin in order to lose weight. Alternatively, the patient may become preoccupied with keeping her blood glucose normal, and lose weight by reducing her dietary intake. In bulimia nervosa, the commonest pattern is omission of insulin, but a small number of patients will try to compensate physiologically for a binge by increasing their insulin. Finally, a subgroup of women may not fit formal criteria for an eating disorder, but manipulate insulin as part of a pre-occupation with controlling their weight.

Despite a lack of detailed outcome studies, the overall impression is that treatment is difficult, and the outcome often disappointing. ${ }^{22,23}$ Previous studies have largely described the problem as it presents to a diabetologist. In order to devise treatments primarily aimed at the eating difficulties, it seems appropriate to approach it in the context of a clinical population of eating-disordered patients.

It is uncertain whether the background factors which lead to eating disorders in a diabetic population are similar to those in nondiabetics, or whether there are more specific features which distinguish the two. If the association between the two disorders is attributable to chance alone, one would expect similar background factors to operate in eating disorders, with or without diabetes mellitus. If, however, diabetes carries an increased risk of developing an eating disorder, mediated, for example, by the increased emphasis on food composition and weight, ${ }^{15}$ then one might expect different patterns of vulnerability to emerge. In order to examine these possibilities, we have carried out a case-control study, using non-diabetic eating-disordered patients from the same clinic as a comparison group.

\section{Methods}

\section{CASES}

A consecutive series of diabetic patients seen at the Maudsley Hospital Eating Disorders Unit between January 1990 and June 1994.

\section{CASE CONTROLS}

Non-diabetic patients, matched for eatingdisorder diagnosis, age of onset, and duration of illness, and seen within the same time period. Age of onset and duration of illness were chosen as these variables are known to affect treatment outcome.

\section{METHODOLOGY}

The study was carried out retrospectively, using a combination of case-notes, assessment sheets, and personal knowledge of the patients by staff of the Unit. Where possible, current state of the diabetes was assessed by one of the authors (MC), working at the Diabetic Unit at
King's College Hospital; otherwise diabetic status was ascertained from the patient's diabetologist, or from entries in the case-notes. At the time of first assessment, all patients had a standard Maudsley history taken.

The following variables were recorded:

\section{Eating disorders}

Diagnosis (past and current), age of onset, duration, body mass index (BMI) (current, highest and lowest), amenorrhoea, weight control methods (vomiting, laxative or diuretic abuse, over-exercising, or others), and treatment history.

\section{Family history}

Eating disorders, depression, alcoholism and other psychiatric illness in a first or second degree relative.

\section{Childhood experiences}

Death of a parent/caregiver; adverse experiences (physical or sexual abuse).

\section{Diabetes}

Type, age of onset, misuse of insulin, and diabetic complications.

\section{STATISTICS}

Comparisons were made using paired $t$-tests, and McNemar or binomial tests for continuous and categorical data, respectively. In addition, because the data set was not always complete for each variable, and because this was an exploratory analysis, we used unpaired $t$-tests and chi-squared or Fisher's exact tests. The former analysis was taken as primary because of the greater power of a paired study.

\section{Results}

Twenty-one patients presented to the Unit between January 1990 and June 1994 with a dual diagnosis of diabetes mellitus and an eating disorder. Case controls were available from the clinic for 17 of these patients, matched for diagnosis, age of onset, and duration of illness. Comparisons between the groups described below refer to these 17 , the remaining four patients were atypical, and will be described separately. A complete set of data was not always available for each patient, and this is indicated where it occurs.

\section{DEMOGRAPHIC DETAILS}

The median (range) age of the cases was 27 (21-46) years, and of the case controls 27 $(20-44)$ years. Of the cases, 14 were single, two were married, and one was separated/divorced. Of the case controls, 11 were single, one was married, and five were separated or divorced.

\section{Eating disorder}

The variables of interest are presented in the table. Eleven in each group were bulimic, five were anorexic, and each contained one obese binge-eater. The only difference between the two groups was in the number who had had previous psychiatric treatment for their eating disorder, which was significantly greater in the 
Table Comparison of the two groups

\begin{tabular}{lcc}
\hline & $\begin{array}{c}\text { Diabetics } \\
(n=17)\end{array}$ & $\begin{array}{c}\text { Controls } \\
(n=17)\end{array}$ \\
\hline $\begin{array}{l}\text { Diagnosis: } \\
\text { bulimia nervosa }\end{array}$ & 11 & 11 \\
anorexia nervosa & 5 & 5 \\
$\quad$ obese binge-eater & 1 & 1 \\
Vomiting & 9 & 10 \\
Laxatives & 8 & 10 \\
Current mean (BMI $\left.k g / \mathrm{m}^{2}\right)$ & $(S E M)$ & $22.8(1.1)$ \\
$\quad$ bulimia nervosa & $22.1(0.7)$ & $16.1(0.7)$ \\
anorexia nervosa & $16.6(0.4)$ & $42.2(1 \mathrm{case})$ \\
$\quad$ obese binge-eater & $44.2(1 \mathrm{case})$ & $6 / 17^{\star}$ \\
Previous treatment & $13 / 16^{\star}$ & $3 / 17$ \\
Death of parent/caregiver & $4 / 14$ & $7 / 17^{\star \star}$ \\
Adverse childhood experience & $1 / 16^{\star \star}$ &
\end{tabular}

${ }^{\star}$ Binomial (2-tailed): $p=0.02 ;$ Chi-squared $=5.4: p=0.02 .{ }^{\star \star B i n o m i a l ~(2-t a i l e d): ~}$ $\mathrm{p}=0.06$; Fisher's exact test: $\mathrm{p}=0.04$

diabetic group (binomial test: $p=0.02$; chisquared (with continuity correction): $p=$ 0.02). The median (range) age of onset of the eating disorder in the diabetic group was 18 (13-30) years, and in the case controls 18 (14-31) years, which reflects clinical norms in an eating-disordered population.

\section{Family history}

There was no significant difference between the two groups in the number who had a family history of eating disorder (four of 17 controls vs two of 13 diabetics), depression (three of 17 controls $v s$ four of 13 diabetics), or alcoholism (four of 17 controls $v$ f four of 12 diabetics).

\section{Childhood experiences}

The two groups did not differ significantly on the incidence of death of a parent/carer. The case-control group, however, had more physical or sexual trauma during childhood (binomial test: $p=0.06$; Fisher's exact test: $p=0.04$ ). Seven of the case-control group were affected, compared to one of the diabetic group. Of these seven, four were sexually abused, five physically abused, and two patients had suffered both forms of abuse. The affected patient with diabetes had suffered physical abuse. Information on childhood trauma was not available for one diabetic patient.

\section{Diabetes}

Results for the matched group of 17 will be presented first.

Matched cases The age of onset (median and range) of diabetes was $15(5-46)$ years. In 12 cases, the onset of diabetes preceded the development of an eating disorder, in one case it occurred around the same time, and in four cases the eating disorder developed first. Only one patient did not require insulin; in this case the eating disorder had preceded the diabetes. All but four of the 16 insulin-dependent patients abused insulin in order to control their weight. Diabetic complications were noted in 11 of the 17 patients, although, even among the six who were complication free, hypoglycaemic attacks were a problem in two cases. The complications included eye disease (seven patients, four of whom required laser treatment), peripheral neuropathy (six patients) autonomic neuropathy (four patients), and necrobiosis lipoidica (one patient). Five women from this group had bone densitometry, and all five scans showed osteoporosis. Two of these patients, aged 26 and 35, had already suffered fractures. Although anorexia nervosa is a wellrecognised risk factor for osteoporosis, it is our clinical impression that it occurs in a more severe form when associated with diabetes.

Atypical cases We were unable to find matched controls for four diabetic cases (see boxes $1-4)$. These were atypical in a number of ways. Two were male; one, aged 65 , was the oldest in the series. The two women, aged 52 and 58, were also older than the average. Three of the four atypical cases were initially non-insulin dependent, although two of these went on to need insulin. Diabetic complications were seen in three patients, and varied from early background retinopathy, to retinal haemorrhages requiring laser treatment.

Case 1

A male, aged 65, lost three stone in weight in the year after his diabetes was diagnosed, from $10 \frac{1}{2}$ to $7 \frac{1}{2}$ stone. He was admitted for investigation of his weight loss but nothing abnormal was found physically. His wife, who had had diabetes mellitus for 30 years and coped well, thought he had taken his dietary instructions to the extreme. A detailed interview revealed his perfectionism and how this affected his diabetes; he had rigidly controlled his diet until he was only eating cereals and fruit. He explained that he had been in the Navy where discipline, and the need to excel at simple chores, were deemed to be important; 'a job is not worth doing unless it can be done well'. He was considered to have an atypical eating disorder in the context of an obsessional personality.

Box 1

Case 2

A female, aged 52, had a 30-year history of insulin dependent diabetes and had developed bulimia nervosa aged 41 . Her weight varied over time between $8 \frac{1}{2}$ and 20 stone. Binge eating began around the time she went to a slimming club and lost $1 \frac{1}{2}$ stone. She would binge on high carbohydrate foods and her overeating was usually precipitated by feeling depressed. $\mathrm{Her}$ depression, in fact, became the main focus of treatment, although her eating disorder remained a problem. Over time, she has had both in- and out-patient treatment, cognitive therapy and psychodynamic treatment. Perhaps surprisingly, the only diabetic complication to date is early background retinopathy.

Box 2 
Case 3

A 32-year-old man presented with bulimia nervosa in the context of diabetes mellitus and polydrug abuse. The eating and drug problems began in his teens, perhaps as a reaction to a very strict upbringing which included physical beatings from his father. As he grew 'big and strong', on comfort food supplied by his mother, the beatings stopped. His weight fluctuated considerably, most often it was around 13 stone, which he was unhappy with, but at one point it went as low as 10 stone. Shortly before his diabetes was diagnosed, aged 18, he weighed 19 stone. He was initially treated with oral hypoglycaemics, but he went on to need insulin. He developed bulimia nervosa in his mid 20s.

Box 3

\section{Case 4}

A 58-year-old woman developed non-insulin dependent diabetes at the age of 40 . At the time, she was moderately overweight (13 stone), but, with the onset of her diabetes, she lost three stone. Bulimic behaviour began in her mid-50s. Her eating disorder and low self-esteem responded well to a course of cognitive behavioural therapy. She was later found to have a growth-hormone-secreting pituitary adenoma. She was treated with pituitarectomy and radiotherapy and her diabetes now requires insulin.

Box 4

\section{Discussion}

This was an exploratory study, guided by our clinical impression that women with diabetes in addition to an eating disorder had a somewhat different course and outcome to that of our usual clinic population. Because our numbers were not large in statistical terms, we ran the risk of a type II error occurring, ie, missing a significant difference where one actually existed. However, in practice, it would have been difficult to conduct a larger study because of the relative infrequency of a dual diagnosis. In fact, apart from the variables highlighted below, no other variables approached statistical significance as discriminators.

A number of interesting points arose from the study. In particular, there were suggestive differences in treatment histories and background factors between the two groups. Differing levels of childhood trauma were found, occurring in seven of the case controls, and in only one of the diabetic group. The incidence of trauma in the case controls was comparable to that seen in our previous study on childhood care in eating disorders in the bulimic subgroup, ${ }^{24}$ where $20 / 76 \quad(26 \%)$ patients suffered childhood sexual abuse, and $20 / 79(25 \%)$ had a history of physical abuse.

The exact mechanisms whereby adverse events in childhood increase vulnerability to the development of an eating disorder is un-

\section{Summary points}

- in the majority of patients, diabetes developed before the eating disorder

- a high incidence of diabetic complications was found in this patient group

- significantly more of the diabetic patients had had previous attempts at treatment for their eating disorder

- the incidence of childhood trauma was lower in the diabetic group

Box 5

known. Striegel-Moore suggests that such events impair the development of a secure identity. ${ }^{25}$ The onset of diabetes in childhood or early adolescence may also damage the developing sense of identity and lead to low selfesteem. In our clinical experience, patients commonly blame themselves for their diabetes, with attributes such as, 'it came about because I secretly rebelled from my parents and had sweets', or 'I thought that if I controlled my diet perfectly, it would go away. I didn't realise I had it for life'. These attributions cause guilt and shame and so are held secretly and unchallenged. One may hypothesise that, other factors being equal, the diagnosis and implications of diabetes provide sufficient difficulty to provoke the onset of an eating disorder.

Significantly more of the diabetic group had had previous treatment, suggesting that treatment is more difficult in this subgroup. Traditional methods, such as straight cognitive behavioural therapy, may be less suitable for diabetic patients. ${ }^{26}$ For example, self-monitoring in the form of a food diary, an ingredient of cognitive behavioural therapy, is resisted by many diabetic patients, who have often spent years monitoring their food intake. The aims of treatment must also be slightly different. The diabetic patient needs to retain control of food and weight.

Eating disorders in diabetes may present less classically, with abuse of insulin as a preferred weight control method, and there is the possibility of diagnostic confusion due to the weight changes associated with poorly controlled diabetes. The four atypical cases are of particular interest as they suggest that groups not usually considered to be at risk, such as women over 40 , as well as males, are vulnerable in the context of diabetes. Whether the increased risk is biological or psychological, or a mixture of both, may not be relevant for treatment, as our patient with a pituitary tumour responded well to a purely psychological approach.

To summarise, we have reported on a series of diabetic patients with eating disorders and compared these to a group of non-diabetic patients from the same clinic. We have found that many diabetic patients referred here had had previous attempts at treatment. A high incidence of diabetic complications was noted, with clear implications, both clinically and economically, for early intervention. Finally, 
the incidence of childhood trauma was lower in our diabetic group, suggesting that perhaps the diabetes itself provides the vulnerability and increases the risk of developing an eating disorder. Early intervention in diabetic clinics may prevent the development of serious eating disorders, thus highlighting the need for

1 Bruch H. Eating disorders: obesity, anorexia, and the person within. New York: Basic Books, 1973; p 357.

2 Russell GFM. Bulimia nervosa: an ominous variant of Russell GFM. Bulimia nervosa: an ominous var
anorexia nervosa? Psychol Med 1979; 9: 429-88.

3 Fairburn CG, Steel JM. Anorexia nervosa in diabetes Fairburn CG, Steel JM. Anorex
mellitus. BMf 1980; 280: 1167-8.

4 Marcus MD, Wing RR. Eating disorders and diabetes. In: Holmes CS, ed. Neuropsychological and behavioral aspects of insulin and non-insulin dependent diabetes. New York: Springer Verlag, 1990; pp 102-721.

5 Lloyd GG, Steel JM, Young RJ. Eating disorders and psychiatric morbidity in patients with diabetes mellitus. Psychother Psychosom 1987; 48: 189-95.

6 Rosemark B, Berne C, Holmgren S, et al. Eating disorders in patients with insulin-dependent diabetes mellitus. $f \mathrm{Cl}$ Psychiatry 1986; 47: 547-50.

7 Striegel-Moore RH, Nicholson TJ, Tamborlane WV. Prevalence of eating disorder symptoms in pre-adolescent and adolescent girls with IDDM. Diabetes Care 1992; 15 and adolesc $1361-8$.

8 Peveler RC, Fairburn CG, Boller I, Dunger D. Eating disorders in adolescents with IDDM: a controlled study. disorders in adolescents with IDD

9 Marcus MD, Wing RR, Jawad A, Orchard TJ. Eating disorder symptomatology in a registry-based sample of disorder symptomatology in a registry-based sample of Eating Disord 1992; 12: 425-30.

10 Meadows GN, Palmer RL, Newball EUM, Kenrick JMT Eating attitudes and disorder in young women: a general practice based survey. Psychol Med 1986; 16: 351-7.

11 Rastam M, Gillberg C, Garton M. Anorexia nervosa in Swedish urban region: a population based study. $\mathrm{Br}$ Psychiatry 1989; 155: 642-6.

12 Patton GC, Johnson-Sabine E, Wood K, Mann AH, Wakeling A. Abnormal eating attitudes in London schoolgirls - a prospective epidemiological study: outcome at twelve month follow-up. Psychol Med 1990; 20: 383-94.

13 Beglin SJ, Fairburn CG. Evaluation of a new instrument for the detection of eating disorders in community samples. Psychiatry Res 1992; 44: 191-201. psychological input into adolescent diabetic clinics.

We wish to acknowledge the statistical advice provided by Dr Graham Dunn, Department of Biostatistics and Computing, Institute of Psychiatry.

14 Stewart DE, Erlick Robinson G, Goldbloom DS, Wright C Infertility and eating disorders. Am $₹$ Obstet Gynecol 1990; 163: 1196-9.

15 Steel JM, Young RJ, Lloyd GG, MacIntyre CCA. Abnormal eating attitudes in young, insulin-dependent diabetics. mal eating attitudes in young, insulin

16 Kendler KS, MacLean C, Neale M, Kessler R, Heath A, Eaves $L$. The genetic epidemiology of bulimia nervosa. $A m \mathcal{F}$ Psychiatry 1991; 148: 1627-37.

17 Bushnell JA, Wells E, Hornblow AR, Oakley-Browne MA, Joyce P. Prevalence of three bulimia syndromes in the general population. Psychol Med 1990; 20: 671-8.

18 Fairburn CG, Beglin SJ. Studies of the epidemiology of bulimia nervosa. Am $f$ Psychiatry 1990; 147: 401-8.

19 Rodin GM, Johnson LE, Garfinkel PE, et al. Eating disorders in female adolescents with insulin-dependen diabetes mellitus. Int f Psychiatry Med 1986-7; 16: 49-57.

20 Rodin G, Craven J, Littlefield C Murray M. Eating disorders and intentional insulin und disorders and inter

21 Steel J. Eating disorders. In: Kelmar CJH, ed. Childhood and adolescent diabetes. Chapman \& Hall, 1994; pp 375-84.

22 Peveler RC, Fairburn CG. Anorexia nervosa in association with diabetes mellitus - a cognitive behavioral approach to with diabetes mellitus - a cognitive behavio

23 Szmukler G. Anorexia nervosa and bulimia in diabetics. $f$ Pzychosom Res 1984; 28: 365-9.

24 Schmidt U, Tiller J, Treasure J. Setting the scene for eating disorders: childhood care, classification and course of illness. Psychol Med 1993; 23: 663-72.

25 Striegel-Moore RH. Etiology of binge eating: a developmental perspective. In: Fairburn CG, Wilson GT, eds. Binge eating: nature, assessment, and treatment. New York: The Guildford Press, 1993; pp 144-72.

26 Peveler RC, Fairburn CG. The treatment of bulimia nervosa in patients with diabetes mellitus. Int f Eating Disord 1992, 11: $45-53$. 